\title{
Current induced torques and interfacial spin-orbit coupling: Semiclassical modeling
}

\author{
Paul M. Haney, ${ }^{1}$ Hyun-Woo Lee, ${ }^{2}$ Kyung-Jin Lee, ${ }^{1,3,4,5}$ Aurélien Manchon, ${ }^{6}$ and M. D. Stiles ${ }^{1}$ \\ ${ }^{1}$ Center for Nanoscale Science and Technology, National Institute of Standards and Technology, Gaithersburg, Maryland 20899-6202, USA \\ ${ }^{2}$ PCTP and Department of Physics, Pohang University of Science and Technology, Kyungbuk 790-784, Korea \\ ${ }^{3}$ Department of Materials Science \& Engineering, Korea University, Seoul 136-713, South Korea \\ ${ }^{4}$ KU-KIST Graduate School of Converging Science \& Technology, Korea University, Seoul 136-713, Korea \\ ${ }^{5}$ University of Maryland, Maryland Nanocenter, College Park, Maryland 20742, USA \\ ${ }^{6}$ Core Labs, King Abdullah University of Science and Technology (KAUST), Thuwal 23955-6900, Saudi Arabia
}

(Received 18 January 2013; published 7 May 2013)

\begin{abstract}
In bilayer nanowires consisting of a ferromagnetic layer and a nonmagnetic layer with strong spin-orbit coupling, currents create torques on the magnetization beyond those found in simple ferromagnetic nanowires. The resulting magnetic dynamics appear to require torques that can be separated into two terms, dampinglike and fieldlike. The dampinglike torque is typically derived from models describing the bulk spin Hall effect and the spin transfer torque, and the fieldlike torque is typically derived from a Rashba model describing interfacial spin-orbit coupling. We derive a model based on the Boltzmann equation that unifies these approaches. We also consider an approximation to the Boltzmann equation, the drift-diffusion model, that qualitatively reproduces the behavior, but quantitatively differs in some regimes. We show that the Boltzmann equation with physically reasonable parameters can match the torques for any particular sample, but in some cases, it fails to describe the experimentally observed thickness dependencies.
\end{abstract}

DOI: 10.1103/PhysRevB.87.174411

PACS number(s): 85.35.-p, 72.25.-b

\section{INTRODUCTION}

Spintronic applications like spin-transfer-torque magnetic random access memory (STT-MRAM) or magnetic domain wall-based devices require advances in materials to reach their full potential. The goal of improving these materials has led to the study of bilayers consisting of ferromagnetic layers and nonmagnetic layers with strong spin-orbit coupling. Recent measurements on such systems have demonstrated efficient switching of magnetic tunnel junctions, ${ }^{1}$ like those used in STT-MRAM, and efficient current-driven domain wall motion. ${ }^{2}$

There are a number of physical processes ${ }^{3}$ in these systems that contribute to the magnetization dynamics as described by the Landau-Lifshitz-Gilbert equation. These include the typical micromagnetic contributions, like interatomic exchange, magnetostatic interactions, magnetocrystalline anisotropy, and damping, as well as the adiabatic and nonadiabatic spin transfer torques $^{4-11}$ that are typically added to account for the coupling between the magnetization and the electrical current flowing through it. In the bilayers of interest here, there are additional contributions that have received extensive attention. These arise from the spin-orbit coupling in the nonmagnetic layer and from the enhanced spin-orbit coupling at the interfaces between layers.

These additional contributions have been modeled in terms of two different pictures. One picture ${ }^{12}$ assumes that the layers are thick and the two layers have their bulk properties. A current flowing through the nonmagnetic layer with strong spin-orbit coupling generates a spin current perpendicular to the interface (the spin Hall effect). ${ }^{13-17}$ When this spin current impinges on the interface, there is a spin transfer torque ${ }^{18-21}$ on the magnetization of the magnetic layer. The details of the torque in this picture are determined by the bulk spin Hall angle in the material with strong spin-orbit coupling and the mixing conductance. The other picture ${ }^{22-25}$ assumes two-dimensional transport that can be described by a Rashba model, similar to those used to describe spin-orbit coupling in two-dimensional electron gases. ${ }^{26}$ The Rashba model gives direct coupling between the magnetization and the flowing current. Both models give qualitatively similar results, that is, torques along the $\mathbf{M} \times(\mathbf{j} \times \hat{\mathbf{z}})$ and $\mathbf{M} \times[\mathbf{M} \times(\mathbf{j} \times \hat{\mathbf{z}})]$ directions, where $\mathbf{M}$ is the magnetization, $\mathbf{j}$ is the in-plane current density, and the interface normal is in the $\hat{\mathbf{z}}$ direction. We refer to the first torque as a fieldlike torque because it has the same form as precessional torque around an effective field in the $-\mathbf{j} \times \hat{\mathbf{z}}$ direction. The second torque has the same form as a damping torque toward a field in that same direction and we refer to it as a dampinglike torque. ${ }^{27}$ We note that the dampinglike torque can also act like an antidamping torque ${ }^{28}$ depending on the details of the magnetization and the current direction.

Both models have strengths and weaknesses. The Rashba model treats the strong spin-orbit coupling at the interfaces between the materials but treats the transport as two dimensional. The layer thicknesses are usually comparable to mean free paths and spin-diffusion lengths, requiring a three-dimensional description of the transport. On the other hand, the spin-Halleffect spin-transfer-torque model treats the three-dimensional aspect of the transport, but ignores any contributions from the modification of the spin-orbit coupling near the interface. The nonmagnetic layer and the magnetic layer affect the electronic structure of each other close to the interface and the interaction can significantly change the spin-orbit coupling there. In particular, the proximity to the ferromagnet can induce a moment in the material with strong spin-orbit coupling and the material with strong spin-orbit coupling can induce a large spin-orbit effect in the ferromagnet. Both effects give a thin layer where the magnetism and the spin-orbit coupling coexist. ${ }^{29}$

Attempts to develop predictive models face the complication that the experimental structures deviate significantly 
from the ideal structures treated theoretically. Experimental indications ${ }^{30,31}$ that interfaces of Co grown on Pt have different properties than interfaces of Pt grown on Co argue strongly that the details of the interface structure are both nontrivial and important. Unfortunately, the interfaces are not well enough characterized to know what types of disorder might be present. There may be significant interdiffusion at the interfaces because, for example, $\mathrm{Pt}$ alloys with Co in the bulk. There is also significant lattice mismatch between the materials. This mismatch could promote thickness fluctuations and dislocation formation. Without measurements of atomic scale structure of the experimental samples, it is impossible to know how important such defects are to the behavior of the system.

Motivated by the uncertainty in the details of the experimental structures and the goal of incorporating the strengths of existing models, we develop simple semiclassical models for these systems. One approach is based on the drift-diffusion equation and the other on the Boltzmann equation. The latter is able to capture the essential physics of the models that have been used so far and provides a test for whether a model based on bulk properties and enhanced spin-orbit coupling at the interface can account for the experimental behavior. We find that this model is general enough to reproduce the torques measured in any single sample for reasonable values of the parameters, but not all samples with a single parametrization. In Sec. II we summarize the experimental evidence for the existence of dampinglike and fieldlike torques. In Sec. III we give the details of the drift-diffusion approach we use and find an analytic expression for the torques in the absence of interfacial spin-orbit coupling. We describe the Boltzmann equation approach we use in Sec. IV. This approach simultaneously treats interfacial spin-orbit coupling and the spin Hall effect in the nonmagnetic layer. Section V compares the analytic result for the drift-diffusion approach to calculations done with the Boltzmann equation and highlights the circumstances where the two differ quantitatively. Section VI gives the results of calculations with both the spin Hall effect and the Rashba interaction showing that the two do not interfere with each other. That is, the interfacial spin-orbit coupling does not significantly modify the torque due to the bulk spin Hall effect. At the same time, it leads to additional torques that are very closely related to those found in the two-dimensional Rashba model calculations.

\section{EXPERIMENTAL RESULTS}

The recent interest in bilayer systems began with a series of experiments by Liu et al. ${ }^{1,32-34}$ and Miron et al. ${ }^{2,35,36}$ The authors of the first set of experiments interpret their results in terms of a dominant dampinglike torque that they attribute to the spin Hall effect. On the other hand, the authors of the second set of experiments interpret their results in terms of both dampinglike and fieldlike torques, which they attribute to the interfacial spin-orbit coupling. The Rashba model is invoked to describe the interfacial spin-orbit coupling.

In case of the spin Hall picture, the spin Hall effect in a nonmagnetic layer injects electrons with one particular spin direction into its adjacent magnetic layer. This interlayer spin current generates the dampinglike torque, which has the same structure as the Slonczewski torque ${ }^{18-21}$ generated by spin-polarized current flowing perpendicular to the layers in magnetic multilayer systems. Liu et al. examine various magnetization dynamics using this picture. In Pt/Py bilayers ${ }^{32}$ they use the spin-torque ferromagnetic resonance technique to show that the spin Hall effect is strong enough to cause magnetic precession. Through resonance line shape analysis, they quantify the spin Hall angle of Pt to be about +0.076 , which is about two orders of magnitude larger than the corresponding value in $n$-doped GaAs. ${ }^{37,38}$ Such a large spin Hall angle makes the spin Hall effect a realistic tool to enhance spin torque efficiency. In their subsequent work ${ }^{1}$ for the $\mathrm{Ta} / \mathrm{Co}_{40} \mathrm{Fe}_{40} \mathrm{~B}_{20}$ bilayer, they report the larger spin Hall angle of -0.12 to -0.15 for $\mathrm{Ta}$, and demonstrate that the spin-Hall-induced dampinglike torque can switch the magnetization in a reliable and efficient way, facilitating the development of magnetic memory and nonvolatile spin logic technologies. A still larger spin Hall angle of $-0.33 \pm 0.06$ is obtained for $\mathrm{W}$ in their more recent experiment. ${ }^{39}$

On the other hand, Miron et al. , $35,36^{\text {explore effects }}$ of the Rashba spin-orbit coupling on $\operatorname{Pt}(3 \mathrm{~nm}) / \mathrm{Co}(0.6$ $\mathrm{nm}) / \mathrm{AlO}_{x}(2 \mathrm{~nm})$. They report ${ }^{35}$ that an in-plane current j flowing through the system enhances or suppresses the nucleation of reversed magnetic domains just like an in-plane transverse magnetic field does. This result is in qualitative agreement with the current-induced transverse effective field ${ }^{40}$ $\mu_{0} \mathbf{H}_{\mathrm{R}}$,

$$
\mu_{0} \mathbf{H}_{\mathrm{R}} \approx \frac{\alpha_{\mathrm{R}}}{2 \mu_{\mathrm{B}} M_{\mathrm{s}}} P(\hat{\mathbf{z}} \times \mathbf{j}),
$$

which is theoretically predicted ${ }^{22-25}$ based on the Rashba spin-orbit coupling of the form $\alpha_{\mathrm{R}}(\mathbf{k} \times \hat{\mathbf{z}}) \cdot \sigma$. Here $M_{\mathrm{S}}$ is the saturation magnetization, $P$ is the spin polarization, and $\mu_{\mathrm{B}}$ is the Bohr magneton. The effective field $\mathbf{H}_{\mathrm{R}}$ generates the fieldlike torque $-\gamma \mathbf{M} \times \mathbf{H}_{\mathrm{R}} \propto-\mathbf{M} \times(\hat{\mathbf{z}} \times \mathbf{j})$, where $\gamma$ is the gyromagnetic ratio. They also examine current-driven domain wall motion ${ }^{35}$ in the same system. Even when the driving current density goes up, the domain wall motion does

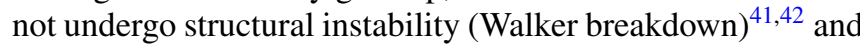
the domain wall velocity increases to $\approx 400 \mathrm{~m} / \mathrm{s}$, which is estimated to be twice as large as the spin angular momentum transfer rate $|\mathbf{j}|\left(P g \mu_{\mathrm{B}}\right) /\left(2 e M_{\mathrm{S}}\right)$, where $g(\approx 2)$ is the gyromagnetic ratio. Conventional adiabatic and nonadiabatic ${ }^{6,8}$ spin transfer torque cannot explain this result. The authors suggest that $\mathbf{H}_{\mathrm{R}}$ suppresses the Walker breakdown and explains the experimental result. Intriguingly, the domain wall in the experiment moves against the electron flow, which is contrary to previous experiments, ${ }^{43-45}$ where domain walls move along the electron flow.

In a later experiment ${ }^{36}$ on the same system, they observe current-driven bipolar switching, which is attributed to a dampinglike torque. Based on the observation that the efficiency of the bipolar switching increases with the magnetic anisotropy of the cobalt layer and the oxidation of the aluminum layer, they argue that the dampinglike torque arises mainly from the Rashba spin-orbit coupling. Calculations ${ }^{3,46-48}$ suggest that the Rashba spin-orbit coupling can give rise to dampinglike torques. In contrast, Liu et al. ${ }^{33}$ conclude that the dampinglike torque in $\mathrm{Pt}(2.0 \mathrm{~nm}) / \mathrm{Co}(0.6 \mathrm{~nm}) / \mathrm{Al}(1.6 \mathrm{~nm})$ arises mainly from the spin Hall effect in $\mathrm{Pt}$ and the Rashba spin-orbit 
coupling contribution is negligible. The latter conclusion is based on their failure to measure a significant current-induced transverse field.

Regardless of its physical origin, the dampinglike torque can significantly enhance ${ }^{47,49}$ the current-driven domain wall velocity since it is either parallel or antiparallel to the nonadiabatic spin transfer torque at the domain wall center. When antiparallel, the domain wall can move against the electron flow, explaining the reversed domain wall direction. ${ }^{2}$ This enhancement mechanism is similar to that in spin valves. ${ }^{50}$

Other groups find additional features. Measurements on $\mathrm{Ta}(1.0 \mathrm{~nm}) / \mathrm{Co}_{40} \mathrm{Fe}_{40} \mathrm{~B}_{20}(1.0 \mathrm{~nm}) / \mathrm{MgO}(2.0 \mathrm{~nm})$ find the transverse field to be about $23 \%$ of the magnitude in Ref. 35. A similar magnitude is reported ${ }^{51}$ for $\operatorname{Pt}(3.0$ $\mathrm{nm}) / \mathrm{Co}(0.6 \mathrm{~nm}) / \mathrm{AlO}_{x}(1.8 \mathrm{~nm})$, but the magnitude appears to be sensitive to layer thicknesses. A systematic thickness dependence study ${ }^{52}$ for the two wedge systems, $\mathrm{Ta}\left(d_{\mathrm{Ta}}\right) / \mathrm{Co}_{20} \mathrm{Fe}_{60} \mathrm{~B}_{20}(1 \mathrm{~nm}) / \mathrm{MgO}(2 \mathrm{~nm})$ and $\mathrm{Ta}(1$ $\mathrm{nm}) / \mathrm{Co}_{20} \mathrm{Fe}_{60} \mathrm{~B}_{20}\left(t_{\mathrm{CoFeB}}\right) / \mathrm{MgO}(2 \mathrm{~nm})$, find that the magnitudes of both the current-induced transverse and longitudinal (related to the dampinglike torque) fields change considerably as a function of both the Ta layer thickness $d_{\mathrm{Ta}}$ and the $\mathrm{Co}_{20} \mathrm{Fe}_{60} \mathrm{~B}_{20}$ layer thickness $t_{\mathrm{CoFeB}}$. Interestingly, the currentdriven domain wall velocity is also reported to be sensitive to layer thicknesses. ${ }^{31}$ Several experiments ${ }^{30,53-55}$ report reversed domain wall motion in ultrathin multilayer systems containing Pt layers.

In addition to the fieldlike and dampinglike torques, which are independent of the gradient of the magnetization, other possibilities that depend on the gradient of the magnetization and the layer structure are allowed by symmetry ${ }^{3}$ and may be important for the dynamics. A recent micromagnetic calculation $^{56}$ suggests that a current-independent torque due to the Dzyaloshinskii-Moriya interaction ${ }^{57}$ may also exist and stabilize a moving domain wall above the nominal Walker-breakdown field. In this paper we consider the currentinduced torques that are independent of the gradient of the magnetization.

\section{DRIFT-DIFFUSION FORMALISM}

To explore possible mechanisms for the torques in these systems, we develop semiclassical models that allow for easy exploration of parameter space. We use a Boltzmann equation approach and the simpler drift-diffusion approach. The Boltzmann equation is better suited to describe in-plane transport but the drift-diffusion approach is simpler and provides a useful language to describe the physics. In this section we describe the drift-diffusion approach and in the next section the Boltzmann equation approach.

The drift-diffusion approach of Valet and Fert ${ }^{58}$ is based on integrating the Boltzmann equation to derive transport equations that depend on the densities and currents. It has had wide success describing current-perpendicular-to-theplane giant-magnetoresistance (GMR), but does not describe current-in-the-plane GMR. It fails because it does not describe the flow of spin currents between the layers when the net current flows in the plane of the layers. This limitation is less important in the bilayer systems of interest here. In materials with strong spin-orbit coupling, like Pt, spin currents do flow perpendicular to the charge current because of the spin Hall effect ${ }^{13-15}$ so that the drift-diffusion approach does qualitatively describe the physics. However, we show in Sec. V that in some parameter regimes, the drift-diffusion approach differs quantitatively from the Boltzmann equation for similar reasons to its qualitative failure for current-in-the-plane GMR.

In the drift diffusion model, spin-dependent scattering leads to a different conductivity for the majority electrons $\sigma^{\uparrow}$ than the minority electrons $\sigma^{\downarrow}$. This difference is parametrized in terms of the spin polarization of the current, defined through $P=$ $\left(\sigma^{\uparrow}-\sigma^{\downarrow}\right) /\left(\sigma^{\uparrow}+\sigma^{\downarrow}\right)$. The drift-diffusion transport equations in the ferromagnet are

$$
\begin{aligned}
\mathbf{j} & =\sigma \nabla \mu-P \sigma \nabla\left(\hat{\mathbf{M}} \cdot \boldsymbol{\mu}^{\mathrm{s}}\right), \\
Q_{i j} & =\frac{\hbar}{2 e} \hat{M}_{j} P \sigma \nabla_{i} \mu-\frac{\hbar}{2 e} \sigma \nabla_{i} \mu_{j}^{\mathrm{s}},
\end{aligned}
$$

where $\mathbf{j}$ is the charge current density, and $\mathbf{Q}$ is the tensor spin current density where the first index is the spatial component and the second index is the spin component. $\mu$ is the electrochemical potential, such that negatively charged electrons diffuse against the gradient giving an overall positive sign for the first term in Eq. (2). Similarly, $\boldsymbol{\mu}^{\mathrm{s}}$ is the spin chemical potential, which is a vector along the direction of the spin accumulation, and the unit vector $\hat{\mathbf{M}}$ is the direction of the magnetization. The minus sign in the second term of Eq. (2) arises because majority electron spins are aligned opposite to the magnetization. These two signs are typical of possible sources of confusion in this subject matter. They arise because the charge on the electron is negative and angular momenta and moments are in opposite directions.

The steady-state continuity equations in the ferromagnet are

$$
\begin{gathered}
\nabla \cdot \mathbf{j}=0, \\
\nabla_{i} Q_{i j}=-\frac{1}{\tau_{\mathrm{ex}}}(\mathbf{s} \times \hat{\mathbf{M}})_{j}-\frac{1}{\tau_{\mathrm{sf}}} s_{j}-\frac{1}{\tau_{\mathrm{dp}}}[\hat{\mathbf{M}} \times(\mathbf{s} \times \hat{\mathbf{M}})]_{j},
\end{gathered}
$$

where the spin accumulation $\mathbf{s}$ is proportional to the spin chemical potential $\mathbf{s}=\mathcal{N}_{\mathrm{s}} \boldsymbol{\mu}^{\mathrm{s}}$, with a constant of proportionality $\mathcal{N}_{\mathrm{s}}$ related to the density of states. The precession time $\tau_{\mathrm{ex}}=\hbar / \Delta$ is related to the exchange splitting $\Delta$ between the magnetization and the spin accumulation. Repeated indices are summed over. The first term on the right-hand side of Eq. (5) is the precession in the exchange field, the second term is the spin-flip scattering that reduces all components of the spin accumulation, and the last term is the dephasing that reduces only the parts of the spin accumulation transverse to the magnetization.

Dephasing occurs when spins arriving at a point have precessed different amounts so that their transverse components tend to cancel. Dephasing can occur from a variety of processes. In the context of spin transfer torques, it can occur from the variation of electron velocities over the Fermi surface, ${ }^{59}$ or from spins precessing at the same rate but arriving at different times due to scattering. ${ }^{60}$ Setting the transverse spin accumulation to zero, as done in earlier Boltzmann equation calculations $^{61}$ and in magnetoelectronic circuit theory, ${ }^{62}$ is equivalent to taking the limit that the dephasing time goes to zero. 
We note that for the systems considered here, the precession time due to the first term Eq. (5) is faster than the scattering time. This situation invalidates the diffusive approximation that is the basis for Eq. (3). However, when we solve the drift-diffusion equations, we use boundary conditions based on magnetoelectronic circuit theory, which set the transverse spin currents and spin accumulations to zero in the ferromagnet. Thus, in practice, this breakdown of the diffusive limit does not affect our results. The Boltzmann equation, which we discuss in the next section, does not suffer from this breakdown.

In the nonmagnetic material, the explicit forms of the charge and spin currents in the drift-diffusion approximation we use $\operatorname{are}^{63}$

$$
\begin{gathered}
\mathbf{j}=\sigma \nabla \mu-\sigma_{\mathrm{SH}}\left(\nabla \times \mu^{\mathrm{s}}\right), \\
Q_{i j}=-\frac{\hbar}{2 e} \sigma \nabla_{i} \mu_{j}^{\mathrm{s}}-\frac{\hbar}{2 e} \sigma_{\mathrm{SH}} \epsilon_{i j k} \nabla_{k} \mu,
\end{gathered}
$$

where $\sigma$ is the conductivity, $\sigma_{\mathrm{SH}}$ is the spin Hall conductivity coupling the spin and charge currents to the charge and spin potentials, and $\epsilon_{i j k}$ is the Levi-Civita symbol. We neglect a term in Eq. (7) that arises from gradients in the spin chemical potential and side-jump scattering, ${ }^{63}$ assuming that its contribution is small.

The torque on the magnetization is given by the torque between the magnetization and the spin accumulation

$$
\mathbf{T}=\frac{\gamma}{\tau_{\mathrm{ex}} M_{\mathrm{s}}} \mathbf{M} \times \mathbf{s}+\frac{\gamma}{\tau_{\mathrm{dp}} M_{\mathrm{s}}^{2}} \mathbf{M} \times(\mathbf{M} \times \mathbf{s}),
$$

where the gyromagnetic ratio $\gamma=g \mu_{\mathrm{B}} / \hbar$ converts from angular momentum (spin density) to magnetization (so $\mathbf{T}$ is a term in the Landau-Lifshitz-Gilbert equation). ${ }^{27}$ The second term captures the torque due to the dephasing of the electron spins as they precess in the exchange field. Combining this equation with the continuity equation (5) relates the torque to the divergence of the spin current. Integrating the resulting expressions over the ferromagnetic layer relates the total torque to the net spin flux. ${ }^{64}$

The currents and densities in each layer are combined through boundary conditions to give a solution for the whole layer. Here we use boundary conditions from magnetoelectronic circuit theory ${ }^{62}$ to derive an analytical expression of spin torque caused by the spin Hall effect in NM|FM bilayer structures where NM has strong spin-orbit. The charge and spin currents satisfy the boundary conditions at the NM|FM boundary $(z=0)$ given by

$$
\begin{aligned}
j_{z}= & \left(G^{\uparrow}+G^{\downarrow}\right) \Delta \mu-\left(G^{\uparrow}-G^{\downarrow}\right) \Delta \boldsymbol{\mu}_{s} \cdot \hat{\mathbf{M}}, \\
\hat{\mathbf{z}} \cdot \mathbf{Q}= & \operatorname{Re}\left[G^{\uparrow \downarrow}\right]\left(2 \Delta \boldsymbol{\mu}_{s} \times \hat{\mathbf{M}}\right) \times \hat{\mathbf{M}}-\operatorname{Im}\left[G^{\uparrow \downarrow}\right]\left(2 \Delta \boldsymbol{\mu}_{s} \times \hat{\mathbf{M}}\right) \\
& -\left(G^{\uparrow}+G^{\downarrow}\right) \Delta \boldsymbol{\mu}_{s}+\left(G^{\uparrow}-G^{\downarrow}\right) \Delta \mu \hat{\mathbf{M}},
\end{aligned}
$$

where $G^{\uparrow}$ and $G^{\downarrow}$ are interface conductances for majority and minority spins, aligned antiparallel and parallel to $\hat{\mathbf{M}}$, respectively, $G^{\uparrow \downarrow}$ is the mixing conductance, $\Delta \mu=\mu(z=$ $+0)-\mu(z=-0)$ is the chemical potential drop over the interface, and $\Delta \mu_{\mathrm{s}}$ is the spin chemical potential drop across the interface.

For simplicity we assume that the dephasing time in Eq. (5) goes to zero, in which case the spin current transverse to the magnetization at the interface is absorbed by the ferromagnet at the interface and gives the torque. We write the interfacial torque in the form

$$
\mathbf{T}=\delta(z) \frac{g \mu_{\mathrm{B}} j_{0}}{2 e}\left[\tau_{\mathrm{d}} \hat{\mathbf{M}} \times(\hat{\mathbf{M}} \times \hat{\mathbf{y}})+\tau_{\mathrm{f}} \hat{\mathbf{M}} \times \hat{\mathbf{y}}\right],
$$

where $\delta(z)$ localizes the torque to the interface at $z=0$. The dimensionless coefficients $\tau_{\mathrm{d}}$ and $\tau_{\mathrm{f}}$ characterize the "dampinglike" and "fieldlike" contributions, respectively. Other terms are possible, as in Ref. 29, but in the present calculations we find these other terms to be negligible for the parameters we consider.

Solving the bulk equations [Eqs. (2)-(8)] the boundary conditions at the interface between the materials [Eq. (9)] with the additional boundary conditions $j_{z}=0$ at $z=0$, and $\mathbf{j}_{s}=0$ at $z=+t_{\mathrm{F}}$ and $z=-t_{\mathrm{N}}$, where $t_{\mathrm{F}}$ and $t_{\mathrm{N}}$ are the thicknesses of FM and NM, respectively, gives two vector components of the torque as in Eq. (10),

$$
\begin{aligned}
\tau_{\mathrm{d}}= & \theta_{\mathrm{SH}} \frac{\left(1-e^{-t / l_{\mathrm{sf}}}\right)^{2}}{1+e^{-2 t / l_{\mathrm{sf}}}} \\
& \times \frac{\left|\tilde{G}^{\uparrow \downarrow}\right|^{2}+\operatorname{Re}\left[\tilde{G}^{\uparrow \downarrow}\right] \tanh ^{2}\left(t / l_{\mathrm{sf}}\right)}{\left|\tilde{G}^{\uparrow \downarrow}\right|^{2}+2 \operatorname{Re}\left[\tilde{G}^{\uparrow \downarrow}\right] \tanh ^{2}\left(t / l_{\mathrm{sf}}\right)+\tanh ^{4}\left(t / l_{\mathrm{sf}}\right)}, \\
\tau_{\mathrm{f}}= & \theta_{\mathrm{SH}} \frac{\left(1-e^{-t / l_{\mathrm{sf}}}\right)^{2}}{1+e^{-2 t / l_{\mathrm{sf}}}} \\
& \times \frac{\operatorname{Im}\left[\tilde{G}^{\uparrow \downarrow}\right] \tanh ^{2}\left(t / l_{\mathrm{sf}}\right)}{\left|\tilde{G}^{\uparrow \downarrow}\right|^{2}+2 \operatorname{Re}\left[\tilde{G}^{\uparrow \downarrow}\right] \tanh ^{2}\left(t / l_{\mathrm{sf}}\right)+\tanh ^{4}\left(t / l_{\mathrm{sf}}\right)},
\end{aligned}
$$

where $\theta_{\mathrm{SH}}=\sigma_{\mathrm{SH}} / \sigma_{\mathrm{N}}$ is the spin Hall angle and $l_{\mathrm{sf}}^{N}$ is the spindiffusion length of NM. The final factor in each expression depends on a scaled mixing conductance

$$
\tilde{G}^{\uparrow \downarrow}=G^{\uparrow \downarrow} \frac{2 l_{\mathrm{sf}} \tanh \left(t / l_{\mathrm{sf}}\right)}{\sigma_{\mathrm{N}}},
$$

where $\sigma_{\mathrm{N}}$ is the conductivity of NM. The length $l_{\mathrm{sf}} \tanh \left(t / l_{\mathrm{sf}}\right)$ ranges from $t$ for small values of $t / l_{\mathrm{sf}}$ to $l_{\mathrm{sf}}$ for large values. We note that both $\tau_{\mathrm{d}}$ and $\tau_{\mathrm{f}}$ do not depend on the magnetization direction. Thus for the case with the spin Hall effect only, the angular dependence of the torque on the magnetization direction is completely determined by the cross products in Eq. (10). These results are independent of the thickness of the ferromagnetic layer because the boundary conditions force the transverse spin current to be zero in the ferromagnet.

To compare with the Boltzmann equation, we compute the mixing conductance for a model in which all of the Fermi surfaces are spherical and the same size, but in which spindependent reflection arises from a spin-dependent $\delta$-function potential at the interface, $V(\mathbf{r})=\left(\hbar^{2} k_{\mathrm{F}} u_{\sigma} / m\right) \delta(z)$ for $\sigma=$ $\uparrow, \downarrow$, where $\uparrow$ and $\downarrow$ refer to majority and minority electrons respectively. ${ }^{65}$ The mixing conductance in Eqs. (11) and (12) is evaluated by the integral ${ }^{62}$

$$
G^{\uparrow \downarrow}=\frac{e^{2}}{h} \int_{\mathrm{FS}} \frac{d^{2} k}{(2 \pi)^{2}}\left(1-r_{\downarrow} r_{\uparrow}^{*}\right),
$$

where FS refers to integrating over the Fermi surface. For the model treated here, the reflection amplitudes are $u_{\sigma} k_{\mathrm{F}} /\left(i k_{z}-u_{\sigma} k_{\mathrm{F}}\right)$, and the mixing conductance becomes

$$
G^{\uparrow \downarrow}=\frac{e^{2} k_{\mathrm{F}}^{2}}{2 \pi h} \int_{0}^{1} d x x\left(1-\frac{u^{\downarrow}}{i x-u^{\downarrow}} \frac{u^{\uparrow}}{-i x-u^{\uparrow}}\right)
$$




$$
\begin{aligned}
= & \frac{e^{2} k_{\mathrm{F}}^{2}}{2 \pi h}\left(\frac{1}{2}+\frac{u^{\uparrow} u^{\downarrow}}{2\left(u^{\uparrow}+u^{\downarrow}\right)}\left[u^{\downarrow} \ln \left(\frac{u^{\downarrow^{2}}}{1+u^{\downarrow^{2}}}\right)\right.\right. \\
& \left.+u^{\uparrow} \ln \left(\frac{u^{\uparrow^{2}}}{1+u^{\uparrow^{2}}}\right)\right]+i \frac{u^{\uparrow} u^{\downarrow}}{2\left(u^{\uparrow}+u^{\downarrow}\right)} \\
& \left.\times\left\{u^{\downarrow}\left[\pi-2 \tan ^{-1}\left(u^{\downarrow}\right)\right]-u^{\uparrow}\left[\pi-2 \tan ^{-1}\left(u^{\uparrow}\right)\right]\right\}\right) .
\end{aligned}
$$

If $u^{\downarrow}>u^{\uparrow}$ as is the case here, both the real and the imaginary parts of the mixing conductance are positive.

\section{BOLTZMANN EQUATION FORMALISM}

The Boltzmann equation is a semiclassical approach based on the approximation that in some small but not too small region of space it is possible to define electron wave packets that have both a well defined momentum and a well defined position. It is related to a density matrix approach which neglects all of the coherence between states with different wave vectors. The basic quantity of interest is the distribution function $f(\mathbf{k}, \mathbf{r})$, which is the probability to find an electron with wave vector $\mathbf{k}$ at position $\mathbf{r}$.

The Boltzmann equation approach developed by Camley and Barnaś ${ }^{66}$ is the simplest model that describes currentin-the-plane GMR. The distribution function accounts for electrons moving in all directions even though the total current only points in a single direction. This generality allows the approach to describe the flow of spins between the layers even though the current flows in the plane of the interfaces.

Reference 61 describes the matrix Boltzmann equation we use in this paper. It is a generalization of the model used to describe current-in-the-plane GMR, ${ }^{66}$ and is based on a simplified model for the electronic structure. We treat all Fermi surfaces as spherical and as having the same Fermi wave vector. This approach ignores the details of the Fermi surfaces, which are undoubtedly important for specific systems, particularly those with strong spin-orbit coupling. However, the scattering mechanisms are both unknown and uncharacterized, so for simplicity it is appropriate to consider models in which scattering rates and other physical processes are parametrized and the details of the electronic structure are neglected. By performing appropriate integrals over the distribution function, the Boltzmann equation can be transformed into a drift-diffusion equation like that discussed in Sec. III. The parametrized processes in the Boltzmann equation then have a simple connection to those in the drift-diffusion equation.

The straightforward generalization of the Boltzmann equation for spin polarized systems is to have separate distribution functions for up and down electrons $f^{\uparrow}$ and $f^{\downarrow}$. This is the approach used by Camley and Barnaś ${ }^{66}$ to model GMR. For systems with spin-orbit coupling or noncollinear magnetizations, in which spins can point in arbitrary directions, there are two related approaches to generalizing the distribution function. In analogy with the density matrix, the distribution function can be generalized to a $2 \times 2$ Hermitian matrix in spin space $f$. Alternatively, the same information can be captured by four real distribution functions $f_{\alpha}$, which are related to $\mathrm{f}$ by

$$
f_{\alpha}=\operatorname{Tr}\left[\sigma_{\alpha} \mathrm{f}\right],
$$

where $\sigma_{\alpha}$ are the identity and the Pauli spin matrices for $\alpha=$ $0, x, y, z$, respectively.

The generalized Boltzmann equation is

$$
\begin{aligned}
& \frac{\partial f_{\alpha}}{\partial t}+\frac{d \mathbf{r}}{d t} \frac{\partial f_{\alpha}}{\partial \mathbf{r}}+\frac{d \mathbf{k}}{d t} \frac{\partial f_{\alpha}}{\partial \mathbf{k}}+\gamma H_{\beta}^{\mathrm{ex}} f_{\gamma} \epsilon_{\alpha \beta \gamma} \\
& ={\frac{d f_{\alpha}}{d t}}_{\text {coll }}\left[f_{\beta}(t, \mathbf{r}, \mathbf{k}, n)\right],
\end{aligned}
$$

where the collision term on the right-hand side depends on all four distribution functions. The last term on the left-hand side describes spin precession in ferromagnetic layers, where the electron spins precess in the exchange field $\mathbf{H}^{\mathrm{ex}}$. The time derivatives of $\mathbf{r}$ and $\mathbf{k}$ are given by

$$
\begin{aligned}
\frac{d \mathbf{r}}{d t} & =\mathbf{v}_{\mathbf{k}, n}, \\
\hbar \frac{d \mathbf{k}}{d t} & =-e \mathbf{E},
\end{aligned}
$$

where $\mathbf{v}_{\mathbf{k}, n}$ is the velocity of the electron and $\mathbf{E}$ is the electric field. For the linearized Boltzmann equation, we make the replacement

$$
f_{\alpha}(\mathbf{k}) \rightarrow f_{\mathrm{eq}}(\epsilon(\mathbf{k})) \delta_{\alpha, 0}+g_{\alpha}(\mathbf{K}) f_{\mathrm{eq}}^{\prime}(\epsilon(\mathbf{k})) .
$$

Upper case $\mathbf{K}$ refers to wave vectors restricted to the Fermi surface. $f_{\mathrm{eq}}^{\prime}$ is the energy derivative of the Fermi function, emulating a Taylor series expansion of the distribution function around equilibrium.

After some standard algebra, the linearized Boltzmann equation can be cast into the form

$$
\begin{aligned}
& {\left[\mathbf{v}_{\mathbf{K}} \frac{\partial g_{\alpha}\left(\mathbf{K}_{i}\right)}{\partial \mathbf{r}}-e \mathbf{E} \cdot \mathbf{v}_{\mathbf{K}} \delta_{\alpha, 0}+\gamma H_{\beta}^{\mathrm{eff}} g_{\gamma}(\mathbf{K}) \epsilon_{\alpha \beta \gamma}\right]} \\
& \quad=-R_{\alpha, \alpha^{\prime}}\left(\mathbf{K}_{i}\right) g_{\alpha^{\prime}}\left(\mathbf{K}_{i}\right)+\int_{\mathrm{FS}} d \hat{\mathbf{K}}_{f} P_{\alpha, \alpha^{\prime}}\left(\mathbf{K}_{i}, \mathbf{K}_{f}\right) g_{\alpha^{\prime}}\left(\mathbf{K}_{f}\right) .
\end{aligned}
$$

The first term on the right-hand side is the scattering out term and the second term is the scattering in term. The former describes collision processes that reduce the occupancy of a state and the latter those that increase it.

In the ferromagnet, where we neglect spin-orbit coupling, the scattering is diagonal in a coordinate system aligned with the magnetization. For the magnetization pointing in a general direction ( $\sin \theta \cos \phi, \sin \theta \sin \phi, \cos \theta)$, the spin-dependent scattering matrix for the scattering out terms is

$$
R_{\alpha, \alpha^{\prime}}=U_{\alpha, \beta}^{\mathrm{T}} R_{\beta}^{\mathrm{diag}} \delta_{\beta, \beta^{\prime}} U_{\beta^{\prime}, \alpha^{\prime}}
$$

The diagonal scattering matrix is

$$
R^{\text {diag }}=\left(R^{\uparrow}, \bar{R}, \bar{R}, R^{\downarrow}\right),
$$

in terms of the majority and minority scattering rate $R^{\uparrow}=$ $1 / \tau^{\uparrow}$ and $R^{\downarrow}=1 / \tau^{\downarrow}$, and the transverse scattering rate is taken to be the geometric mean of the spin-dependent scattering 


$$
\begin{aligned}
\bar{R}= & 1 / \sqrt{\tau^{\uparrow} \tau^{\downarrow}} \text {. The transformation matrix is } \\
U= & \left(\begin{array}{cccc}
1 / \sqrt{2} & 0 & 0 & 1 / \sqrt{2} \\
0 & 1 & 0 & 0 \\
0 & 0 & 1 & 0 \\
1 / \sqrt{2} & 0 & 0 & -1 / \sqrt{2}
\end{array}\right)\left(\begin{array}{cccc}
1 & 0 & 0 & 0 \\
0 & \cos \theta & 0 & -\sin \theta \\
0 & 0 & 1 & 0 \\
0 & \sin \theta & 0 & \cos \theta
\end{array}\right) \\
& \times\left(\begin{array}{cccc}
1 & 0 & 0 & 0 \\
0 & \cos \phi & -\sin \phi & 0 \\
0 & \sin \phi & \cos \phi & 0 \\
0 & 0 & 0 & 1
\end{array}\right) .
\end{aligned}
$$

The first matrix transforms between the majority/minority description on one hand and the Cartesian description that is used in the rest of the calculations. The second two matrices rotate the coordinate system.

The scattering in terms are similar. For these terms, where spin-orbit coupling does not play a role, $P_{\alpha, \alpha^{\prime}}$ is independent of wave vector and equal to $R_{\alpha, \alpha^{\prime}}$. There is an additional contribution from spin-flip scattering of the form $R^{\mathrm{sf}} \delta_{\alpha, \alpha^{\prime}}(1-$ $\left.\delta_{\alpha, 0}\right)$ in terms of the spin-flip scattering rate $R^{\text {sf }}=1 / \tau_{\text {sf }}$. The last factor restricts the scattering to the spin distribution functions and not the charge function.

In the nonmagnet, the spin-independent scattering is included through a term of the form $R^{\mathrm{N}} \delta_{\alpha, \alpha^{\prime}}$ in terms of the scattering rate $R^{\mathrm{N}}=1 / \tau$, and spin-flip scattering through a term of the form $R^{\mathrm{Nsf}} \delta_{\alpha, \alpha^{\prime}}\left(1-\delta_{\alpha, 0}\right)$ in terms of the spin-flip scattering rate $R^{\mathrm{Nsf}}=1 / \tau_{\text {sf }}$.

Both the size of the spin Hall effect and its underlying mechanism are controversial. The theory for the spin Hall effect is related to that for the anomalous Hall effect, a subject that has been controversial for decades. ${ }^{67}$ Measurements of the spin Hall angle (the ratio of spin Hall and charge conductivities) for various materials span a range of values. Part of the variation may result from the sensitivity of the extraction of the spin Hall angle from experimental data to other material parameters needed to model the experiments. ${ }^{34}$ Measurements ${ }^{1}$ show that the spin Hall effect in Ta is bigger and of the opposite sign of that in $\mathrm{Pt}$, in agreement with previous calculations. ${ }^{68}$ The agreement between these trends in theory and experiment argues for an intrinsic origin of the effect. However, the calculated spin Hall conductivity for Pt appears to be approximately an order of magnitude too small in comparison to the measured value. Spin Hall angles of approximately the right order of magnitude have been computed ${ }^{69}$ for the extrinsic contributions of various impurities in $\mathrm{Cu}$ and $\mathrm{Au}$.

With this uncertainty in the mechanism for the spin Hall effect, we use the form of scattering appropriate for the extrinsic skew scattering contribution for computational simplicity. In the Boltzmann equation we include skew scattering as described by Engel et $a l .{ }^{38}$ but generalize their results to include scattering that leads to the inverse spin Hall effect in addition to the scattering that gives rise to the spin Hall effect. These scattering terms connect the current with a perpendicular spin current and vice versa. Both our approach and the earlier work ${ }^{38}$ neglect the scattering processes that couple spin currents to spin currents moving in other directions. Such a process contribute to spin relaxation, which we include as a phenomenological spin-flip scattering process. These terms are related to the terms neglected in Eq. (7).
Spin-orbit scattering is more complicated than the scattering processes described above because the scattering rates depend on the initial and final momenta. Engel et al. ${ }^{38}$ give the contribution to the collision integral as

$$
\begin{aligned}
\frac{d \mathbf{f}}{d t}= & \frac{n_{i} \hbar k_{\mathrm{F}}}{m^{*}} \sum_{\mathbf{k}_{\mathrm{f}}} \frac{d \sigma}{d \Omega}\left[\mathrm{f}\left(\mathbf{k}_{i}\right)-\mathrm{f}\left(\mathbf{k}_{f}\right)\right] \\
= & n_{i} \sum_{\mathbf{k}_{\mathrm{f}}} \frac{\hbar k}{m^{*}}\left\{I(\varphi)\left[\mathrm{f}\left(\mathbf{k}_{i}\right)-\mathrm{f}\left(\mathbf{k}_{f}\right)\right]\right. \\
& \left.-I(\varphi) S(\varphi) \boldsymbol{\sigma} \frac{\mathbf{k}_{i} \times \mathbf{k}_{f}}{\left|\mathbf{k}_{i} \times \mathbf{k}_{f}\right|}\left[f_{0}\left(\mathbf{k}_{i}\right)+f_{0}\left(\mathbf{k}_{f}\right)\right]\right\},
\end{aligned}
$$

where $\varphi$ is the angle between $\mathbf{k}_{i}$ and $\mathbf{k}_{f}$. This form is based on assuming that the spin-orbit scattering is weak and only keeping quantities lowest order in the spin-flip scattering. We follow this approximation but also include the scattering that gives the inverse spin Hall effect. We assume that $I(\varphi)$ is a constant and that $S(\varphi)=S\left|\mathbf{k}_{i} \times \mathbf{k}_{f}\right|$, where $S$ is now a constant. Then, in our notation, we have

$$
\begin{aligned}
\frac{d f_{\alpha}}{d t}= & n_{i} \sum_{\mathbf{k}_{f}} \frac{\hbar k}{m^{*}}\left\{I\left[f_{\alpha}\left(\mathbf{k}_{i}\right)-f_{\alpha}\left(\mathbf{k}_{f}\right)\right]-I S n_{\alpha}\left[f_{0}\left(\mathbf{k}_{i}\right)\right.\right. \\
& \left.\left.+f_{0}\left(\mathbf{k}_{f}\right)\right]+I S \delta_{\alpha, 0} n_{\alpha^{\prime}}\left[f_{\alpha^{\prime}}\left(\mathbf{k}_{i}\right)+f_{\alpha^{\prime}}\left(\mathbf{k}_{f}\right)\right]\right\} \\
= & \sum_{\mathbf{k}_{f}}\left[-I S n_{\alpha} f_{0}\left(\mathbf{k}_{f}\right)+I S \delta_{\alpha, 0} n_{\alpha^{\prime}} f_{\alpha^{\prime}}\left(\mathbf{k}_{f}\right)\right]
\end{aligned}
$$

where $\mathbf{n}=\mathbf{k}_{i} \times \mathbf{k}_{f}$ or $n_{\alpha}=\epsilon_{\alpha, \beta, \gamma} k_{i \beta} k_{f \gamma}$. The terms containing $\delta_{\alpha, 0}$ are the additional terms that give the inverse spin Hall effect. In the second step we have dropped the isotropic part because it is simply another contribution to the isotropic scattering. Finally, we absorbed the velocity and the impurity density factor into the scattering rate. Translating to the notation we have been using gives

$$
P_{\alpha, \alpha^{\prime}}=P S\left[k_{i \beta} k_{f \gamma} \epsilon_{\alpha, \beta, \gamma} \delta_{\alpha^{\prime}, 0}-k_{i \beta} k_{f \gamma} \epsilon_{\alpha^{\prime}, \beta, \gamma} \delta_{\alpha, 0}\right] .
$$

The scattering out contribution is zero because $\sum_{\mathbf{k}_{f}} n_{\alpha}=0$.

While the overall structure of the Boltzmann equation approach is the same as that published in Ref. 61, there are some differences. One difference is the treatment of dephasing. In a ferromagnet, spins on different parts of the Fermi surface precess at different rates and travel with different velocities. These differences, combined with scattering between different parts of the Fermi surface, cause the precessing spins to rapidly become out of phase with each other. ${ }^{59}$ In Ref. 61 the transverse spin accumulation and current are forced to zero in the ferromagnet to account for this dephasing of the transverse spin population. Here we allow for transverse spin accumulation in the ferromagnet but build in rapid spin precession and explicitly account for the processes that cause dephasing. The simplified model of the Fermi surfaces that we use can lead to underestimation of dephasing processes. We have tested this approximation by adding an explicit dephasing term. While such a term quantitatively changes the spin accumulation in the ferromagnet, we find that it does not change the calculated torques.

Given the scattering matrices, we find the general solutions of the Boltzmann equation in each layer using the techniques described in Ref. 61. These are matched together at the 
interface through boundary conditions described below and subjected to diffuse or specular boundary conditions at the outer interfaces.

The equality of the Fermi surfaces would also allow for perfect transmission of electrons across the interface between the materials. In the Boltzmann equation we include spindependent reflection by the addition of a spin-dependent sheet potential ( $\delta$ function) at the interface. Choosing the strength of this $\delta$ function allows us to tune the spin-dependent interface resistance to any arbitrary value. ${ }^{70}$ In the driftdiffusion approach, the spin-dependent reflection becomes a spin-dependent interface resistance or conductance as used in the closely related circuit theory, ${ }^{62}$ see Eq. (15).

An important difference with the previously published ${ }^{61}$ formalism for the Boltzmann equation is the inclusion of spin-orbit coupling at the interface. The Rashba interaction is included through an additional term in the interface potential

$$
V(\mathbf{r})=\frac{\hbar^{2} k_{\mathrm{F}}}{m} \delta(z)\left[u_{0}+u_{\mathrm{ex}} \boldsymbol{\sigma} \cdot \hat{\mathbf{m}}+u_{\mathrm{R}} \boldsymbol{\sigma} \cdot(\hat{\mathbf{k}} \times \hat{\mathbf{z}})\right],
$$

where the interface is in the $\hat{\mathbf{z}}$ direction at $z=0, u_{0}$ is the spinindependent part of the potential, $u_{\mathrm{ex}}$ is the spin-dependent part of the potential that gives rise to spin-dependent reflection, $u_{\mathrm{R}}$ is the Rashba contribution, with $\mathbf{k}$ being the wave vector of an electron scattering from the interface, $k_{\mathrm{F}}$ is the Fermi wave vector, and $m$ is the electron mass. This additional term captures the form of spin-orbit coupling that is allowed for the simple electronic structure assumed here. For more realistic band structures, the form would be much more complicated. Unfortunately, it is difficult to compare $u_{\mathrm{R}}$ with the $\alpha_{\mathrm{R}}$ used in previous publications. Doing so requires a procedure for reducing the Hamiltonian for a three-dimensional system to one for a two-dimensional system.

We note that the Rashba contribution to spin-orbit coupling is frequently discussed alongside the Dresselhaus contribution. The former arises from inversion symmetry breaking due to the interface and that latter due bulk inversion asymmetry. Most heavy transition metals form structures that preserve bulk inversion symmetry, so the Dresselhaus contribution can be neglected.

In Eq. (28), the last two terms can be combined to give a wave vector dependent field direction $\hat{\mathbf{u}}(\mathbf{k})$ and strength $u_{\text {eff }}(\mathbf{k})$ such that $u_{\text {eff }}(\mathbf{k}) \hat{\mathbf{u}}(\mathbf{k})=u_{\mathrm{ex}} \hat{\mathbf{m}}+u_{\mathrm{R}} \hat{\mathbf{k}} \times \hat{\mathbf{z}}$. With respect to this direction, the majority and minority transmission and reflection amplitudes are

$$
\begin{gathered}
T=\frac{i k_{z} / k_{\mathrm{F}}}{i k_{z} / k_{\mathrm{F}}-\left(u_{0} \pm u_{\mathrm{eff}}\right)}, \\
R=\frac{u_{0} \pm u_{\mathrm{eff}}}{i k_{z} / k_{\mathrm{F}}-\left(u_{0} \pm u_{\mathrm{eff}}\right)} .
\end{gathered}
$$

Since both the magnitude and phase of the transmission and reflection amplitudes are different for the majority and minority spin components, an electron spin oriented along some arbitrary direction undergoes a finite rotation when transmitted or reflected. This approach keeps the full coherence between all components of the spin during scattering from the interface.

A part of the torque on the electron spin is due to the coupling to the exchange field and a part due to the spin-orbit coupling (Rashba contribution). The reaction torque on the magnetization can be computed from the exchange coupling between the spin density at the interface and the exchange field

$$
\mathbf{T}=\delta(z) \frac{\gamma}{M_{\mathrm{s}}}\left(\frac{\hbar k_{\mathrm{F}} u_{\mathrm{ex}}}{m}\right) \mathbf{s} \times \mathbf{M},
$$

where the spin density $\mathbf{s}$ is calculated from the incoming wave function and the transmission amplitudes. Since the potential is proportional to a $\delta$ function, the torque density diverges but is finite when integrated over a finite thickness.

The treatment we use for the interfacial spin-orbit coupling in the Boltzmann equation does not generalize easily to the drift-diffusion equation because there are no wave vector dependent quantities in that model. It may be possible to define a generalization of the conductance matrix used in the magnetoelectronic circuit theory. In typical usage, the longitudinal spin components couple to each other and the transverse spin components couple to each other, but the longitudinal and transverse spin components do not couple. With the Rashba interaction included, all spin components would couple to all others.

The Boltzmann equation approach differs quite significantly from the approach used in which the system is modeled with a two-dimensional Rashba model. In the Boltzmann equation approach, electron spins get kicked when they pass through the interface, but they spend no time "in" the interface. In the Rashba model, the entire system is the interface so the electrons (and spins) are "in" the interface at all times. In that case, there is a spin accumulation that builds up in the interface. The spin accumulation gives rise to the strong fieldlike torque found in these models. In spite of this difference, we show below that the both approaches give qualitatively similar torques.

The remaining differences with the calculations described in Ref. 61 are related to differences in geometry. The earlier work treated charge current flow perpendicular to the plane and here we treat charge current flow in the plane. In the former case electrons flow through the outer interface into and out of the leads. Here the outer interfaces reflect any incident electrons and can do so specularly, diffusely, or some combination of both.

\section{SPIN HALL EFFECT PLUS SPIN TRANSFER TORQUE}

In this section we describe the behavior of the model in the absence of spin-orbit coupling at the interface. In this limit, the spin Hall effect in the nonmagnetic layer generates a spin Hall current that propagates perpendicular to the interface with spins pointed perpendicular to both the interface normal and the direction of the current. When this spin current hits the interface with the ferromagnet, angular momentum is transferred from the flowing spins to the magnetization as is typical for spin transfer torques in magnetic multilayers. ${ }^{18,19,21}$

This process is shown in Fig. 1 based on calculations done with the Boltzmann equation described in Sec IV. Parameter choices are given in Table I. These have been chosen to approximately have values appropriate for $\mathrm{Co} / \mathrm{Pt}$ bilayers with vacuum on either side. These parameters are either input parameters, calculated numerically based on the input parameters, or determined analytically from them. In the last case, the evaluated expression is given in the table. 

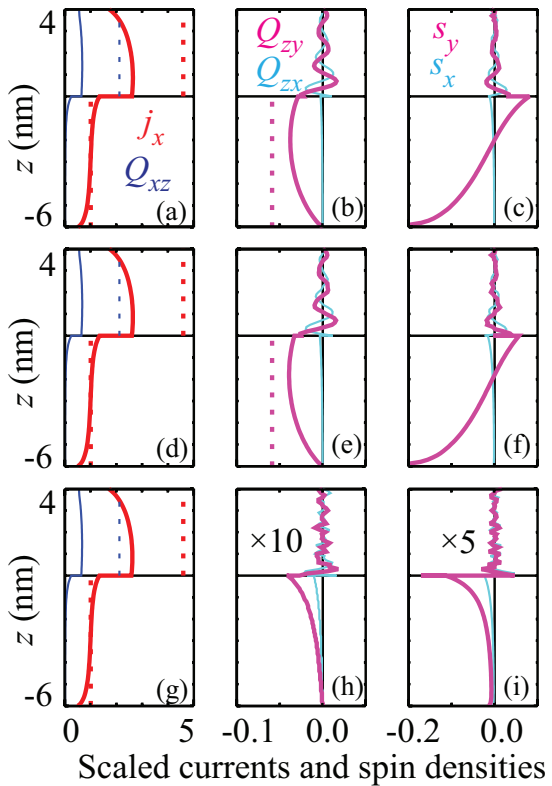

FIG. 1. (Color online) Currents, spin currents, and spin accumulations. The left panels [(a), (d), and (g)] show the current density (heavy lines) $j_{x}$, which is flowing in the plane of the sample and the spin current $Q_{x z}$ (lighter lines), flowing in the $x$ direction with spins aligned with the magnetization in the $z$ direction. The dotted lines indicate the bulk values. All currents and spin currents are dimensionless; currents are scaled by the bulk current in the nonmagnet and spin currents are scaled by the bulk current in the nonmagnet and an additional factor of $\hbar / 2 e$. The spin densities are scaled by the same two factors and $v_{\mathrm{F}}$. The middle panels [(b), (e), and (h)] show the spin currents $Q_{z y}$ (heavy lines) and $Q_{z x}$ (lighter lines), flowing perpendicular to the layers ( $z$ direction) with spins pointing perpendicular to the magnetization, i.e., the $x$ and $y$ directions. The right panels [(c), (f), and (i)] show the accumulation of spin perpendicular to the magnetization $s_{y}$ (heavy lines) and $s_{x}$ (lighter lines). The top panels $[(a)-(c)]$ are for the case in which there is no interfacial spin-orbit coupling, the bottom panels [(g)-(i)] for the case with interfacial spin-orbit coupling $u_{\mathrm{R}}=0.04$ and no spin Hall effect in the nonmagnet, and the middle panels [(d)-(f)] for the case when both are present. In (h) and (i) the spin accumulations have been scaled by the indicated factors.

Figure 1 shows the currents, spin currents, and spin densities for a $4 \mathrm{~nm}$ ferromagnetic layer coupled to a $6 \mathrm{~nm}$ nonmagnetic layer with the interface at $z=0$. Figure 1(a) shows the distribution through the thickness of the films of the current flowing in the plane of the film (in the $x$ direction). The current is greater in the ferromagnetic layer because it has a higher conductivity than the nonmagnetic layer for this choice of parameters. The current is suppressed close to the outer boundaries because we assume that the scattering from those interfaces is completely diffuse. In fact, the ferromagnetic layer is not thick compared to the mean free paths, so the current is suppressed through the thickness of the film. The spin current with spins aligned with the magnetization ( $z$ direction) and moving in the plane also reduced from the bulk value, in fact more so than the current, so the polarization of the current is reduced from the bulk value. At the interface between the two materials, the current is enhanced in the lower conductivity layer due to electrons entering from the higher conductivity
TABLE I. Default parameter values. Parameters for the ferromagnet $(\mathrm{F})$ are chosen to be roughly those for Co as in Ref. 71 and those for the nonmagnet $(\mathrm{N})$ to be roughly those for Pt as in Ref. 34 . $\lambda=v_{\mathrm{F}} \tau$ is a mean free path and $\ell^{\mathrm{sf}}$ is a spin-diffusion length. The rest of the parameters are defined in the text.

\begin{tabular}{lll}
\hline \hline$\lambda_{\mathrm{N}}$ & input & $2.43 \mathrm{~nm}$ \\
$\lambda_{\mathrm{N}}^{\mathrm{sf}}$ & input & $14.7 \mathrm{~nm}$ \\
$\lambda_{\mathrm{sH}}$ & input & $11.8 \mathrm{~nm}$ \\
$\lambda_{\mathrm{F}}^{\uparrow}$ & input & $16.25 \mathrm{~nm}$ \\
$\lambda_{\mathrm{F}}^{\downarrow}$ & input & $6.01 \mathrm{~nm}$ \\
$\lambda_{\mathrm{F}}^{\mathrm{sf}}$ & input & $3280 \mathrm{~nm}$ \\
$\lambda_{\text {ex }}$ & input & $0.258 \mathrm{~nm}$ \\
$k_{\mathrm{F}}$ & input & $16 \mathrm{~nm}^{-1}$ \\
$u_{0}$ & input & 0.42645 \\
$u_{\mathrm{ex}}$ & input & 0.20055 \\
$u_{\mathrm{R}}$ & input & 0.04 \\
$\theta_{\mathrm{SH}}$ & computed & -0.059 \\
$\ell_{\mathrm{N}}^{\mathrm{sf}}$ & computed & $2.57 \mathrm{~nm}$ \\
$\ell_{\mathrm{F}}^{\text {sf }}$ & computed & $69.3 \mathrm{~nm}$ \\
$\sigma_{\mathrm{N}}$ & $\frac{e^{2}}{h} \frac{2 \lambda_{\mathrm{N}}}{3 \pi^{2}} \pi k_{\mathrm{F}}^{2}$ & $0.005 \mathrm{~nm}$ \\
$\rho_{\mathrm{N}}$ & $1 / \sigma_{\mathrm{N}}$ & $20 \mu \Omega \mathrm{cm}^{-1}$ \\
$\sigma_{\mathrm{F}}$ & $\frac{e^{2}}{h} \frac{\lambda_{\mathrm{F}}^{\uparrow}+\lambda_{\mathrm{F}}^{\downarrow}}{3 \pi^{2}} \pi k_{\mathrm{F}}^{2}$ & $0.02 \mathrm{~nm}-1 \Omega^{-1}$ \\
$\rho_{\mathrm{F}}$ & $1 / \sigma_{\mathrm{F}}$ & $5 \mu \Omega \mathrm{cm}^{14}$ \\
$\operatorname{Re}\left[G^{\uparrow \downarrow}\right]$ & Eq. $(15)$ & $5.94 \times 10^{14} \Omega^{-1} \mathrm{~m}^{-2}$ \\
$\operatorname{Im}\left[G^{\uparrow \downarrow}\right]$ & Eq. $(15)$ & $0.86 \times 10^{14} \Omega^{-1} \mathrm{~m}^{-2}$ \\
\hline \hline
\end{tabular}

layer, and the current is reduced in the higher conductivity layer.

This modification of the current near the interface is not captured by a drift-diffusion model. It is one source of the quantitative disagreement between the models as discussed below.

Figure 1(b) shows the two components of the spin current with spins aligned perpendicular to the magnetization and moving perpendicular to the plane of the film. In the nonmagnetic layer this is due to the spin Hall effect. The spin current is zero at the lower boundary, which is both impenetrable and has no spin-flip scattering. It increases to close to its bulk value at the interface between the nonmagnet and the ferromagnet. Inside the nonmagnetic layer, the spin current is a competition between the spin Hall current and a diffusive spin current from the spin accumulation, seen in Fig. 1(c), that builds up due to the impenetrability of the outer interface. Figures $1(\mathrm{~d})-1(\mathrm{i})$ show calculations with interfacial spin-orbit coupling included and are discussed in Sec. VI.

The prefactor in Eq. (10) is based on $j_{0}$, which is the "bulk" current density in the nonmagnetic layer, that is $j_{0}=\sigma_{\mathrm{N}} E$ where $E$ is the applied electric field. This choice seems to be that typically made in analyses of experiments even though the total current is all that is directly measurable. The rest of the factors convert from current density to magnetization torque density $\dot{\mathbf{M}}$. This choice makes sense in analyzing experiments in terms of the spin Hall effect because the torque is driven by the current density in the nonmagnet. However, for thin films, there can be important corrections due to the outer boundaries and the interface with the ferromagnet. These corrections are shown in Fig. 2 for a variety of thicknesses for the two layers. The average current density is reduced by the 


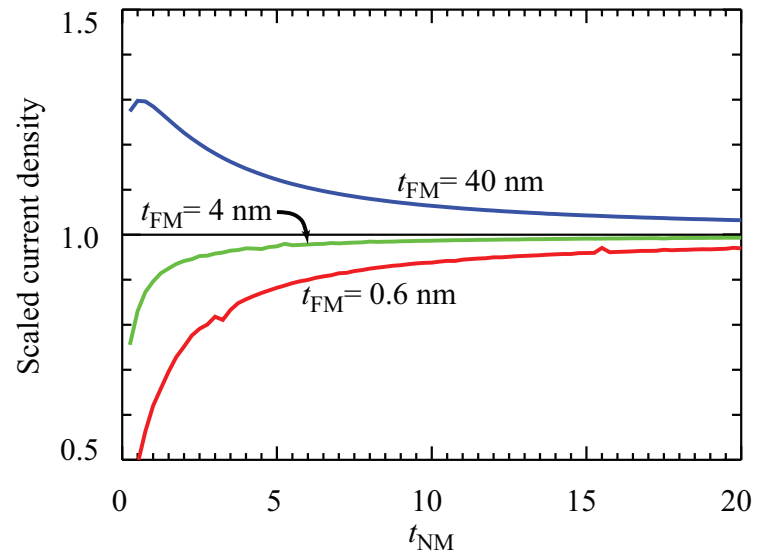

FIG. 2. (Color online) Average current density in the nonmagnetic layer. For three thicknesses of the ferromagnetic layer, the average current density in the nonmagnetic layer is shown scaled by the bulk value as a function of the thickness of the nonmagnetic layer.

diffuse scattering assumed at the outer boundary of the layer, but is increased by the (assumed) higher conductivity of the ferromagnetic layer when that layer is thick enough. In the drift-diffusion approach, there are no such corrections.

For this model, with no interfacial spin-orbit coupling, the spin transfer torque is determined solely by the transverse spin current, ${ }^{59}$ just outside the magnetic layer. Since neither the majority transmission probability is zero nor the minority reflection probability is one, some of the transverse spin current is reflected. The reflected spin current is seen in the reduction of the transverse spin current close to the interface. Some of the transverse spin current is absorbed right at the interface and some is transmitted into the ferromagnet. In the ferromagnet, spin components transverse to the magnetization rapidly precess as they traverse the layer, as seen in the oscillations in Fig. 2(b). Furthermore, they dephase as they traverse the layer as can be seen by the decay of the transverse spin current in the ferromagnet in Fig. 2(b).

The dominant spin transfer torque arises from the absorption of the incident transverse spin current either at the interface or in the ferromagnet. However, not all of the current is absorbed, and some is rotated into the $x$ component of the spin current on reflection. The rotation gives rise to a small fieldlike torque. These torques are shown in Fig. 3 as a function of the thickness of the nonmagnetic layer.

Figure 3 shows the dampinglike and fieldlike torques calculated with both the Boltzmann equation approach ${ }^{72}$ and the drift-diffusion approach. The drift-diffusion approach is given in Eqs. (11) and (12). Both approaches give the same behavior as a function of the thickness of the nonmagnetic layer. Because the spin Hall current in the nonmagnetic layer is suppressed when the layer is thin, as seen in Fig. 1, the torque is reduced when the layer thickness is less than a few spin-diffusion lengths, which for this set of parameters is $\ell_{\mathrm{N}}^{\mathrm{sf}}=2.5 \mathrm{~nm}$.

For thick layers, the value saturates, but does not saturate to the spin Hall angle $\theta_{\mathrm{SH}}$ as might be expected. Equation (11) shows that for the drift-diffusion model, the saturation value depends on the ratio $2 \ell_{\mathrm{N}}^{\mathrm{sf}} \operatorname{Re}\left[G^{\uparrow \downarrow}\right] / \sigma_{\mathrm{N}}$. When this ratio is small,

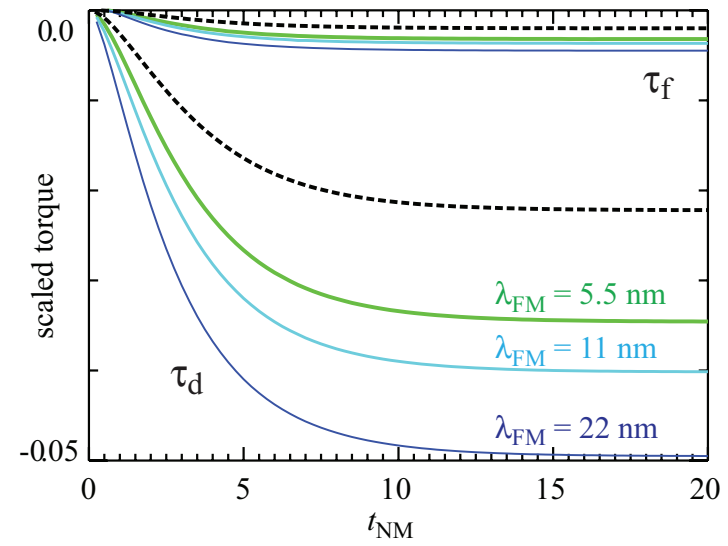

FIG. 3. (Color online) Torques as a function of nonmagnetic layer thickness. The solid curves are the full Boltzmann equation calculation and the dashed curves give the analytic approximation based on the drift-diffusion model and the circuit theory. The more negative curves show the fieldlike torque $\tau_{\mathrm{f}}$ and those closer to zero show the dampinglike torque $\tau_{\mathrm{d}}$. For both torques, the Boltzmann results have been calculated for three different mean free paths (labeled on the dampinglike torques) in the ferromagnet.

the saturation value is reduced from $\theta_{\mathrm{SH}}$ and the Boltzmann calculation and the drift-diffusion calculation saturate to different values. When that ratio is large, the drift-diffusion and Boltzmann equation results agree. However, a large value of this ratio is not physically realistic for systems with strong spin-orbit coupling. The mixing conductance depends mainly on the area of the Fermi surface in the nonmagnet (as a reminder our calculations assume the same Fermi surface for all materials), but does so in the same way that the conductivity does (see the expression in Table I), so it is difficult to increase the ratio by changing the mixing conductance. It is possible to decrease the conductivity by increasing the nonspin-flip scattering, but this also decreases the spin-diffusion length. For the default parameters we consider (see Table I) the value of this ratio is about 0.7 .

Equation (11) also shows that the torque calculated with the drift-diffusion approach is independent of the details of the ferromagnetic layer, depending only on the mixing conductance. The results for the Boltzmann equation, for which we do not have analytic results, do depend on the details of the ferromagnetic layer as seen in Fig. 3 for different values of the mean free path in the ferromagnet. When the mean free path is long so that the conductivity in the ferromagnetic layer is much greater than that in the nonmagnetic layer, the current near the interface in the nonmagnet is increased (see Fig. 1) giving a greater spin Hall current. Another difference is that the only length scale for variation in the drift-diffusion approach is the spin-diffusion length. There are many more length scales in the Boltzmann equation approach (see Table I) and these turn out to play a non-negligible role when $\ell_{\mathrm{N}}^{\mathrm{sf}} \operatorname{Re}\left[g^{\uparrow \downarrow}\right] / \sigma_{\mathrm{N}}$ is not large. The deviation between the results of the Boltzmann equation calculations and those found from the drift-diffusion equation should provide a note of caution for the extraction of physical parameters, like the spin Hall angle, from comparisons between experiment and the drift-diffusion equation. 
We conclude that without additional spin-orbit coupling at the interface between the two materials, three-dimensional transport models predict a torque that is predominantly dampinglike but has a minor fieldlike contribution. In the parameter range we have studied, the torque is always well described by the combination of these two forms. The driftdiffusion approach qualitatively captures the physics but can quantitatively fail in physically relevant parameter regimes.

\section{INCLUSION OF INTERFACIAL RASHBA COUPLING}

The results in Ref. 29 show that there is an interfacial region with significant spin-orbit coupling and exchange splitting. In this section we model that overlap region by adding a Rashba term to the energy at the interface, see Eq. (28). We find that this additional term primarily leads to a fieldlike torque and that as long as it is not too strong it does not significantly modify the torques due to the spin Hall effect.

Previously, this region has been treated by two-dimensional calculations in which the electronic structure is modified by the competition between the Rashba interaction and the exchange interaction. ${ }^{22-25}$ Typically, the Rashba interaction and the exchange interaction are taken to be very different in magnitude so that the Fermi surfaces remain essentially circular. The spin eigendirections on the Fermi surfaces are tied to the wave vector so that the nonequilibrium occupation due to a current flow give rise to a net spin accumulation that is not aligned with the magnetization. This net transverse spin density generates a fieldlike exchange torque on the magnetization.

In the Boltzmann equation approach that we use, the Rashba interaction modifies the boundary conditions for the distribution functions at the interface, see Eqs. (28)-(30). Note that the Boltzmann equation used here treats all spin channels coherently with each other, including in the matching of the distribution functions at the interface. The resulting torque is very similar to what is found in the two-dimensional calculations. Depending on the details of the parameters, the transmission probability is, on average, either greater or lesser for spins aligned with $\hat{\mathbf{z}} \times \mathbf{j}$ than for those in other directions. The spin density at the interface is determined by the transmission probabilities and the incident fluxes. The bias in the transmission probabilities favors a net spin polarization aligned with $\hat{\mathbf{z}} \times \mathbf{j}$, very similar to the behavior found in the two-dimensional treatments. Then, through Eq. (31), there is a fieldlike torque.

The effect of introducing the Rashba term on the current distribution is shown in Fig. 1. Unfortunately, the spin densities at the interface are obscured by the approximations of the Boltzmann equation. In this approach we assume that electrons on different parts of the Fermi surface are incoherent with each other. However, the matching conditions for the distribution functions across the interface are found through coherent scattering calculations. Once the scattering states are used to construct the matching conditions, the coherence between the states with different wave vectors (incoming and outgoing states) is neglected, while the full spin coherence is maintained. As a result, for each electron the spin density at the interface is equal to the incident amplitude times the transmission probability $|T|^{2}$, which is identical to $|1+R|^{2}$ because the

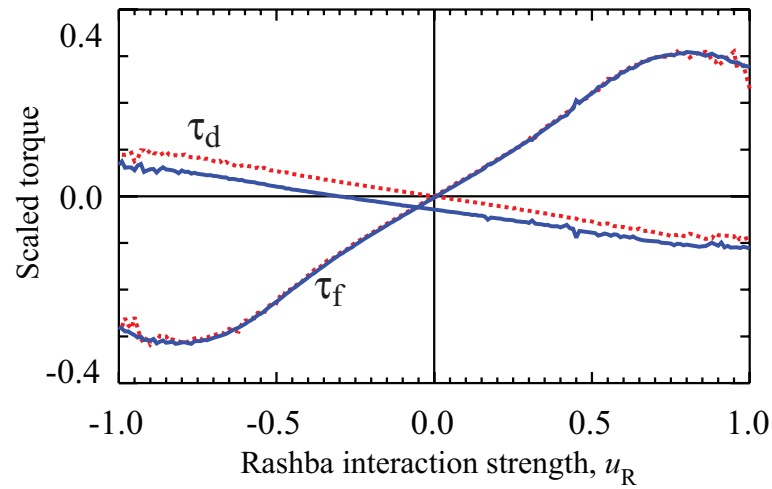

FIG. 4. (Color online) Scaled torques as a function of the interfacial Rashba interaction. The solid curves are calculated with a bulk spin Hall effect and the dotted curves without. The nonmagnetic layer is $6 \mathrm{~nm}$ thick and the ferromagnetic layer is $4 \mathrm{~nm}$. The dampinglike and fieldlike torques are labeled with $\tau_{\mathrm{d}}$ and $\tau_{\mathrm{f}}$, respectively. The jitter is due to numerical instabilities.

wave function is continuous across the interface. However, immediately outside the interface, the incident and reflected states are no longer treated as coherent, on one side of the interface the spin density is proportional to $|T|^{2}$ and on the other $1+|R|^{2}$. Since there are electrons incident from both sides, the spin density at the interface is not equal to the spin density on either side.

In Fig. 1 we have used a strength of the Rashba interaction that gives a torque that is comparable to that found from the spin Hall effect. However, this interaction only has a small effect on the spin currents and accumulations. We find that the spin currents for systems with both the spin Hall effect and the interfacial Rashba interaction can be simply and accurately approximated as follows. The currents in the plane [Figs. 1(a), $1(\mathrm{~d})$, and $1(\mathrm{~g})$ ] are essentially the same in all three systems. For a system with both the spin Hall effect and the Rashba interaction, the transverse spin currents perpendicular to the plane [Fig. 1(e)] are a sum of the transverse spin currents found in systems with one effect or the other [Figs. 1(b) and 1(h)]. The same holds true for the resulting torques, the torques for systems with both effects are approximately the sum of the torques found in the systems with one effect or the other.

The approximate independence of the torques due to the spin Hall effect and the interfacial Rashba interaction is illustrated in Fig. 4 as a function of the strength of the interfacial Rashba interaction. There are contributions to both the fieldlike and dampinglike torques that increase linearly with the Rashba interaction strength up to large values of the torques. Eventually, the transmission probabilities get so low, see Eq. (30), that all electrons are reflected and the torques go to zero. Comparing calculations done with and without the spin Hall effect shows that the interfacial coupling has very little effect on the torque from the spin Hall effect, particularly for small values of the Rashba interaction strength. Without a priori knowledge of the parameters of the system, particularly the spin-diffusion length, the spin Hall angle, and the interfacial spin-orbit coupling, a wide variety of combinations of dampinglike and fieldlike torques are possible. 

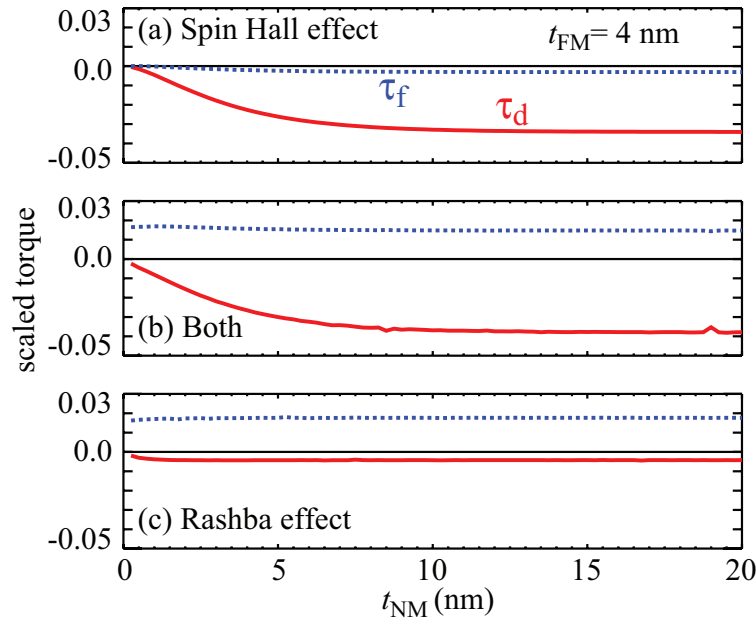

FIG. 5. (Color online) Dimensionless torque components as a function of the thickness of the nonmagnetic layer. (a) The torques in the absence of the Rashba contribution from the interfacial spin-orbit coupling. (c) The torques in the absence of the spin Hall effect in the nonmagnet. (b) The torques with both present. In each part, the solid lines show the dampinglike torque and the dotted lines the fieldlike torque.

Figure 5 shows the torques as a function of the thickness of the nonmagnetic layer. The torques due to the spin Hall effect largely depend exponentially on the nonmagnetic layer thickness divided by the spin-diffusion length. As can be seen from the analytic solution, for very small thicknesses there are corrections such that the torque is not strictly exponential. The exponential variation has been used ${ }^{34}$ to extract the spindiffusion length for particular systems. It is interesting to note that for this simple model, the sign of the product $\tau_{\mathrm{d}} \tau_{\mathrm{f}}$ is opposite in the two limiting cases, the spin Hall effect only case [Fig. 5(a)] and the Rashba effect only case [Fig. 5(c)].
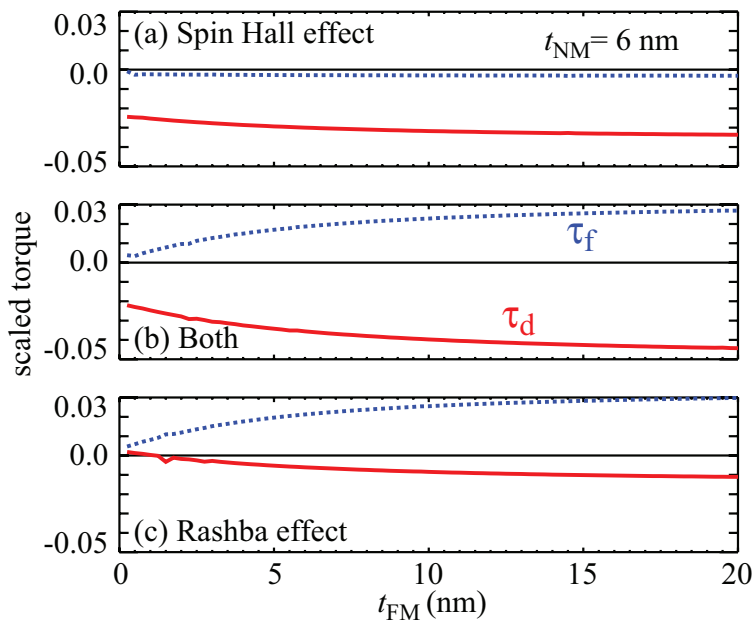

FIG. 6. (Color online) Dimensionless torque components as a function of the thickness of the ferromagnetic layer. (a) The torques in the absence of the Rashba contribution from the interfacial spin-orbit coupling. (c) The torques in the absence of the spin Hall effect in the nonmagnet. (b) The torques with both present. In each part, the solid lines show the dampinglike torque and the dotted lines the fieldlike torque.
Figure 6 shows the torque as a function of the ferromagnetic thickness. As expected from the analytic solution that is displayed in Fig. 3, the torque due to the spin Hall effect only depends weakly on the thickness of the ferromagnetic layer. The variation is largely due to the variation in the current in the nonmagnetic layer due to the presence (and variation) of the ferromagnetic layer. The torque due to the Rashba effect depends more strongly on the ferromagnetic thickness, but not nearly so strongly as is seen in experiment where changing the thickness by a single atomic layer can have a profound effect on the torque.

This discrepancy suggests that the thickness dependence seen in experiment is likely due to physics beyond the scope of the model presented here. One possibility is that the strength of the Rashba interaction could depend sensitively on the thickness of the ferromagnetic layer. The sensitivity could arise from changes in the electronic structure of the interface or even changes in the structure of the interface. Since the lattice mismatch is so large, it is conceivable that the structure evolves rapidly as the layer is made thicker.

\section{SUMMARY}

In this article we have developed semiclassical models for electron and spin transport in bilayer nanowires with a ferromagnetic layer and a nonmagnetic layer with strong spinorbit coupling. We use a Boltzmann equation approach, based on a simplified electronic structure and also a simpler driftdiffusion model. The drift-diffusion framework qualitatively describes the physics of these systems and provides a useful language to discuss their behavior. However, it quantitatively disagrees with the Boltzmann equation to which it is an approximation.

The differences between the results found from the Boltzmann equation and those from the drift-diffusion calculation arise for a couple of reasons. One reason is related to the failure of the drift-diffusion calculation in other cases of in-plane transport. While currents consist of electrons moving in all directions, in the drift-diffusion approximation, that motion in all (three-dimensional) directions is averaged, leaving a single direction for the current. A consequence of this averaging is that the model misses the injection of current and spin current moving parallel to the interface from one layer to the other. In the case of current-in-the-plane GMR, the lack of injected spin currents flowing from one layer to the other eliminates any current-in-the-plane GMR. In the present case, the approximation misses the injection of parallel current across the interfaces. The injection (or reduction) of the current flow in the plane of the interface can change the resulting torque by a factor of 2 or more.

These models provide a framework that naturally includes both the torques due to the bulk spin Hall effect and the spin transfer torque and the torques due to the interfacial spin-orbit coupling. These two torques are the current induced torques that arise from spin-orbit coupling and which are independent of the gradient of the magnetization. The models we treat are qualitatively similar to previous models for the spin-Hallinduced torque but differ substantially from the models used to describe the Rashba torque. Those latter models are based on a two-dimensional treatment of the transport that gives rise 
to a current induced spin accumulation and a predominantly fieldlike torque. The Boltzmann equation approach includes the interfacial spin-orbit coupling in the boundary conditions of a three-dimensional transport calculation. Nevertheless, this approach gives very similar results to the two-dimensional calculations. The interfacial spin-orbit coupling gives rise to a torque that is predominantly fieldlike. Depending on the specific parameters appropriate for a particular system, either the fieldlike torque or the dampinglike torque may dominate.

While the experimental situation is still controversial, there is experimental evidence that both a dampinglike torque and in some systems a fieldlike torque play an important role in the dynamics. The model developed here through the Boltzmann equation captures the physics for both. Unfortunately, it is difficult to make the model predictive rather than explanatory. While many of the transport parameters are known for thick films, they are likely to change significantly in thin films. In fact, many vary with varying thicknesses of the films. This model does capture the variation with the thickness of the nonmagnetic layer, but does not describe the rapid variation with ferromagnetic film thickness found in some systems. This behavior, coupled with the variation of behavior with the order of growth, suggests to us that structural aspects of the samples vary with thickness or growth order. Examples of process that might contribute to this variation include, interdiffusion, strain relief, or grain size.

\section{ACKNOWLEDGMENT}

Professor K.-J. Lee acknowledges support under the Cooperative Research Agreement between the University of Maryland and the National Institute of Standards and Technology Center for Nanoscale Science and Technology, Award 70NANB10H193, through the University of Maryland.
${ }^{1}$ L. Liu, C.-F. Pai, Y. Li, H. W. Tseng, D. C. Ralph, and R. A. Buhrman, Science 4, 555 (2012).

${ }^{2}$ I. M. Miron, T. Moore, H. Szambolics, L. D. Buda-Prejbeanu, S. Auffret, B. Rodmacq, S. Pizzini, J. Vogel, M. Bonfim, A. Schuhl, and G. Gaudin, Nat. Mater. 10, 419 (2011).

${ }^{3}$ E. van der Bijl and R. A. Duine, Phys. Rev. B 86, 094406 (2012).

${ }^{4}$ L. Berger, J. Appl. Phys. 3, 2156 (1978); 3, 2137 (1979).

${ }^{5}$ L. Berger, J. Appl. Phys. 55, 1954 (1984).

${ }^{6}$ S. Zhang and Z. Li, Phys. Rev. Lett. 93, 127204 (2004).

${ }^{7}$ G. Tatara and H. Kohno, Phys. Rev. Lett. 92, 086601 (2004).

${ }^{8}$ A. Thiaville, Y. Nakatani, J. Miltat, and Y. Suzuki, Europhys. Lett. 69, 990 (2005).

${ }^{9}$ Y. Tserkovnyak, A. Brataas, and G. E. W. Bauer, J. Magn. Magn. Mater., 320, 1282 (2008).

${ }^{10}$ G. Tatara, H. Kohno, and J. Shibata, Phys. Rep. 468, 213 (2008).

${ }^{11}$ J. Shibata, G. Tatara, and H. Kohno, J. Phys. D 44, 384004 (2011).

${ }^{12}$ K. Ando, S. Takahashi, K. Harii, K. Sasage, J. Ieda, S. Maekawa, and E. Saitoh, Phys. Rev. Lett. 101, 036601 (2008).

${ }^{13}$ M. I. D'yakonov and V. I. Perel', JETP Lett. 13, 467 (1971).

${ }^{14}$ J. E. Hirsch, Phys. Rev. Lett. 83, 1834 (1999).

${ }^{15}$ S. F. Zhang, Phys. Rev. Lett. 85, 393 (2000).

${ }^{16}$ J. Sinova, D. Culcer, Q. Niu, N. A. Sinitsyn, T. Jungwirth, and A. H. MacDonald, Phys. Rev. Lett. 92, 126603 (2004).

${ }^{17}$ S. Murakami, N. Nagaosa, and S. C. Zhang, Science 301, 1348 (2003).

${ }^{18}$ J. C. Slonczewski, J. Magn. Magn. Mater. 159, L1 (1996).

${ }^{19}$ L. Berger, Phys. Rev. B 54, 9353 (1996).

${ }^{20}$ M. D. Stiles and J. Miltat, Top. Appl. Phys. 101, 225 (2006).

${ }^{21}$ D. C. Ralph and M. D. Stiles, J. Magn. Magn. Mater. 320, 1190 (2007).

${ }^{22}$ K. Obata and G. Tatara, Phys Rev. B 77, 214429 (2008).

${ }^{23}$ A. Manchon and S. Zhang, Phys. Rev. B 78, 212405 (2008).

${ }^{24}$ A. Manchon and S. Zhang, Phys. Rev. B 79, 094422 (2009).

${ }^{25}$ A. Matos-Abiague and R. L. Rodriguez-Suarez, Phys. Rev. B 80, 094424 (2009).

${ }^{26}$ Yu. A. Bychkov and E. I. Rashba, JETP. Lett. 39, 78 (1984).
${ }^{27}$ Strictly speaking, a torque acts on an angular momentum and has units of angular momentum per time. Here we use "torques" to describe contributions to the Landau-Lifshitz-Gilbert equation, $\dot{\mathbf{M}}=-\gamma \mu_{0} \mathbf{M} \times \mathbf{H}+\alpha \hat{\mathbf{M}} \times \dot{\mathbf{M}}+\mathbf{T}$. As such, they have units of magnetization per time $\left[\mathrm{A} \mathrm{m}^{-1} \mathrm{~s}^{-1}\right]$ instead of energy [J].

${ }^{28}$ V. E. Demidov, S. Urazhdin, E. R. J. Edwards, M. D. Stiles, R. D. McMichael, and S. O. Demokritov, Phys. Rev. Lett. 107, 107204 (2011).

${ }^{29}$ J.-H. Park, C. H. Kim, H.-W. Lee, and J. H. Han, Phys. Rev. B 87, 041301 (2013); P. M. Haney (private communication).

${ }^{30}$ R. Lavrijsen, P. P. J. Haazen, E. Mur, J. H. Franken, J. T. Kohlhepp, H. J. M. Swagten, and B. Koopmans, Appl. Phys. Lett. 100, 262408 (2012).

${ }^{31}$ S. S. P. Parkin (unpublished).

${ }^{32}$ L. Liu, T. Moriyama, D. C. Ralph, and R. A. Buhrman, Phys. Rev. Lett. 106, 036601 (2011).

${ }^{33}$ L. Liu, O. J. Lee, T. J. Gudmundsen, D. C. Ralph, and R. A. Buhrman, Phys. Rev. Lett. 109, 096602 (2012).

${ }^{34}$ L. Liu, R. A. Buhrman, and D. C. Ralph, arXiv:1111.3702.

${ }^{35}$ I. M. Miron, G. Gaudin, S. Auffret, B. Rodmacq, A. Schuhl, S. Pizzini, J. Vogel, and P. Gambardella, Nature Materials 9, 230 (2010).

${ }^{36}$ I. M. Miron, K. Garello, G. Gaudin, P.-J. Zermatten, M. V. Costache, S. Auffret, S. Bandiera, B. Rodmacq, A. Schuhl, and P. Gambardella, Nature (London) 476, 189 (2011).

${ }^{37}$ Y. K. Kato, R. C. Myers, A. C. Gossard, and D. D. Awschalom, Science 306, 1910 (2004).

${ }^{38}$ H.-A. Engel, B. I. Halperin, and E. I. Rashba, Phys. Rev. Lett. 95, 166605 (2005).

${ }^{39}$ C.-F. Pai, L. Liu, Y. Li, H. W. Tseng, D. C. Ralph, and R. A. Buhrman, Appl. Phys. Lett. 101, 122404 (2012).

${ }^{40}$ The corresponding equation (1) in Ref. 35 is larger than Eq. (1) in our paper by factor 2. Equation (1) in Ref. 35 is quoted from the theoretical work, ${ }^{23}$ which however contains a factor 2 error. When this error is corrected, it results in Eq. (1) in our paper.

${ }^{41}$ G. S. D. Beach, C. Nistor, C. Knutson, M. Tsoi, and J. L. Erskine, Nat. Mater. 4, 741 (2005).

${ }^{42}$ N. L. Schryer and L. R. Walker, J. Appl. Phys. 45, 5406 (1974). 
${ }^{43}$ A. Yamaguchi, T. Ono, S. Nasu, K. Miyake, K. Mibu, and T. Shinjo, Phys. Rev. Lett. 92, 077205 (2004).

${ }^{44}$ M. Yamanouchi, D. Chiba, F. Matsukura, and H. Ohno, Nature (London) 428, 539 (2004).

${ }^{45}$ M. Hayashi, L. Thomas, C. Rettner, R. Moriya, Y. B. Bazaliy, and S. S. P. Parkin, Phys. Rev. Lett. 98, 037204 (2007).

${ }^{46}$ X. Wang and A. Manchon, Phys. Rev. Lett. 108, 117201 (2012).

${ }^{47}$ K.-W. Kim, S. M. Seo, J. Ryu, K.-J. Lee, and H.-W. Lee, Phys. Rev. B 85, 180404 (2012).

${ }^{48}$ D. A. Pesin and A. H. MacDonald, Phys. Rev. B 86, 014416 (2012).

${ }^{49}$ S.-M. Seo, K.-W. Kim, J. Ryu, H.-W. Lee, and K.-J. Lee, Appl. Phys. Lett. 101, 022405 (2012).

${ }^{50}$ A. V. Khvalkovskiy, K. A. Zvezdin, Ya. V. Gorbunov, V. Cros, J. Grollier, A. Fert, and A. K. Zvezdin, Phys. Rev. Lett. 102, 067206 (2009).

${ }^{51}$ U. H. Pi, K. W. Kim, J. Y. Bae, S. C. Lee, Y. J. Cho, K. S. Kim, and S. Seo, Appl. Phys. Lett. 97, 162507 (2010).

${ }^{52}$ J. Kim, J. Sinha, M. Hayashi, M. Yamanouchi, S. Fukami, T. Suzuki, S. Mitani, and H. Ohno, Nat. Mat. 12, 240 (2013).

${ }^{53}$ J.-C. Lee, K.-J. Kim, J. Ryu, K.-W. Moon, S.-J. Yun, G.-H. Gim, K.-S. Lee, K.-H. Shin, H.-W. Lee, and S.-B. Choe, Phys. Rev. Lett. 107, 067201 (2011).

${ }^{54} \mathrm{~T}$. Ono (unpublished).

${ }^{55}$ S. Emori, U. Bauer, S.-M. Ahn, E. Martinez, and G. S. D. Beach, arXiv:1302.2257.

${ }^{56}$ A. Thiaville, S. Rohart, É. Jué, V. Cros, and A. Fert, Europhys. Lett. 100, 57002 (2012).

${ }^{57}$ I. E. Dzialoshinskii, Sov. Phys. JETP 5, 1259 (1957); T. Moriya, Phys. Rev. 120, 91 (1960).

${ }^{58}$ T. Valet and A. Fert, Phys. Rev. B 48, 7099 (1993).

${ }^{59}$ M. D. Stiles and A. Zangwill, Phys. Rev. B 66, 014407 (2002).

${ }^{60}$ S. Zhang, P. M. Levy, and A. Fert, Phys. Rev. Lett. 88, 236601 (2002).

${ }^{61}$ J. Xiao, A. Zangwill, and M. D. Stiles, European Phys. J. B 59, 415 (2007).

${ }^{62}$ A. Brataas, Y. V. Nazarov, and G. E. W. Bauer, Phys. Rev. Lett. 84, 2481 (2000); A. Brataas, G. E. W. Bauer, and P. J. Kelly, Phys. Rep. 427, 157 (2006)

${ }^{63}$ R. V. Shchelushkin and A. Brataas, Phys. Rev. B 72, 073110 (2005); 73, 169907(E) (2006).

${ }^{64}$ If there is no coupling of angular momentum into the lattice (spin-orbit coupling or spin-flip scattering) it is straightforward to relate Eq. (8) to the divergence of the spin current. In the ferromagnet, where there is spin-flip scattering but no other spinorbit coupling, the corrections to torque being simply the divergence of the spin current can be found from Eq. (5). For the components perpendicular $(\perp)$ to the magnetization, the torque is

$$
\begin{aligned}
& \mathbf{T}=-\gamma(1-\xi \beta)(\nabla \cdot \mathbf{Q})_{\perp}+\gamma \beta \hat{\mathbf{M}} \times(\nabla \cdot \mathbf{Q}), \\
& \beta=\frac{\tau_{\mathrm{ex}}}{\tau_{\mathrm{sf}}} \frac{1}{1+\xi^{2}}, \quad \xi=\frac{\tau_{\mathrm{ex}}}{\tau_{\mathrm{dp}}}+\frac{\tau_{\mathrm{ex}}}{\tau_{\mathrm{sf}}},
\end{aligned}
$$

where $(\nabla \cdot \mathbf{Q})_{\perp}=-\hat{\mathbf{M}} \times[\hat{\mathbf{M}} \times(\nabla \cdot \mathbf{Q})]$ is the component of the divergence of the spin current that is perpendicular to the magnetization. Equation (32) indicates that the spin torque is in general not simply given by the divergence of the spin current but possesses an additional component $\hat{\mathbf{M}} \times \boldsymbol{\nabla} \cdot \mathbf{Q}$ arising from the presence of spin relaxation. For the parameter set used here, $\beta$ is small and this correction is negligible.

${ }^{65}$ Reference 62 gives the mixing conductance as

$$
G^{s,-s}=\frac{e^{2}}{h} \int_{\mathrm{FS}} \frac{d^{2} k}{(2 \pi)^{2}}\left(1-r_{s} r_{-s}^{*}\right) .
$$

Here "up" and "down" spins are defined with respect to the magnetization, so that $s=1$ corresponds to a minority electron with its spin parallel and moment antiparallel to the magnetization.

${ }^{66}$ R. E. Camley and J. Barnaś, Phys. Rev. Lett. 63, 664 (1989).

${ }^{67}$ E. M. Pugh and N. Rostoker, Rev. Mod. Phys. 25, 151 (1953); C. M. Hurd, The Hall Effect in Metals and Alloys (Plenum, New York, 1972); N. A. Sinitsyn, J. Phys.: Condens. Matter 20, 023201 (2008); N. Nagaosa, J. Sinova, S. Onoda, A. H. MacDonald, and N. P. Ong, Rev. Mod. Phys. 82, 1539 (2010).

${ }^{68}$ T. Tanaka, H. Kontani, M. Naito, T. Naito, D. S. Hirashima, K. Yamada, and J. Inoue, Phys. Rev. B 77, 165117 (2008).

${ }^{69}$ M. Gradhand, D. V. Fedorov, P. Zahn, and I. Mertig, Phys. Rev. Lett. 104, 186403 (2010).

${ }^{70}$ M. D. Stiles and D. R. Penn, Phys. Rev. B 61, 3200 (2000).

${ }^{71}$ J. Bass and W. P. Pratt, J. Magn. Magn. Mater. 200, 274 (1999).

${ }^{72}$ The Boltzmann equation calculations reported in Fig. 3 expand the distribution function in a grid of 8 polar angles and 16 azimuthal angles, or 128 total points. Increasing the number of grid points to 16 polar angles and 20 azimuthal angles (320 total points) gives a root mean square difference between the calculations of less than $1 \times 10^{-4}$. 